\title{
Dietary Quality Indices Modify the Effects of Apolipoprotein B Polymorphisms on Cardio- Metabolic Risk Factors in type 2 Diabetes Mellitus: A Cross-Sectional Study
}

\section{Elmira Karimi}

Tehran University of Medical Sciences School of Nutritional Sciences and Dietetics https://orcid.org/0000-0001-7871-2313

\section{Gity Sotoudeh}

Tehran University of Medical Sciences School of Nutritional Sciences and Dietetics

Masoumeh Rafiee

Isfahan University of Medical Sciences

Fariba Koohdani ( $\sim$ fkoohdan@tums.ac.ir)

Tehran University of Medical Sciences

\section{Research article}

Keywords: Apolipoprotein B polymorphism, Dietary index, cardio-metabolic, type 2 diabetes mellitus.

Posted Date: January 20th, 2021

DOI: https://doi.org/10.21203/rs.3.rs-150352/v1

License: (c) (i) This work is licensed under a Creative Commons Attribution 4.0 International License.

Read Full License 


\section{Abstract}

Background: We tried to identify the interaction between dietary quality indices and apolipoprotein B Ins/Del and EcoR1 polymorphisms on cardio-metabolic risk factors in patients with type 2 diabetes mellitus (T2DM).

Methods: This cross-sectional study recruited 700 adults with T2DM who visited diabetes referral centers in Tehran. The genotypes of Ins/Del and EcoR1 single nucleotide polymorphisms (SNP) were explored via polymerase chain reaction (PCR). Dietary quality index-international (DQI-I), healthy eating index-2015 (HEI-2015) and dietary phytochemical index (DPI) were calculated by semi-quantitative food frequency questionnaire (FFQ). Lipid, inflammatory, oxidative, hormonal and anthropometric variables were determined by standard methods.

Results: We observed a significant interaction between DQI-I and Ins/De/SNP on leptin ( $P=0.02$ for crude model and $\mathrm{P}=0.01$ for adjusted model (for age, physical activity, gender, smoking and alcohol intake) and 8-iso-prostaglandin F2 a (8-iso-PGF2a) ( $\mathrm{P}=0.01$ for both models), DPI and EcoR1 SNP on total cholesterol (TC) ( $\mathrm{P}=0.03$ for both models) and between Ins/DelSNP and HEI-2015 on interleukin-18 (IL-18) ( $\mathrm{P}=0.02$ for crude model and $\mathrm{P}=0.03$ for adjusted model). Furthermore, in crude but not in adjusted model there were close to meaningful interactions between EcoR1 SNP and DQII on total antioxidant capacity (TAC) $(\mathrm{P}=0.06)$ and between EcoR1 SNP and HEI-2015 on serum leptin $(\mathrm{P}=0.05)$ and superoxide dismutase (SOD) $(p=0.05)$ levels.

Conclusion: For the first time, our finding indicated that the association between DQI-I, HEI-2015 and DPI with IL-18, TC, leptin and 8-iso-PGF2a in patients with T2DM might be dependent on Ins/Del and EcoR1 variants in $A p o B$ gene.

\section{Background}

Type 2 diabetes mellitus (T2DM) is a pandemic disease associated to insulin resistance, beta-cell dysfunction and hyperglycemia (1). Epidemiological studies reported that worldwide prevalence of diabetes mellitus (DM) was about 451 million in 2017 with most being T2DM. This global prevalence is postulated to be as much as 693 million by 2045 (2). Majority of T2DM patients present with physiological and biochemical abnormalities including elevated oxidative stress and inflammatory status $(3,4)$. Hyperglycemia can induce oxidative stress via over-secretion of advanced glycation end-products (AGEs), polyols and PKC $\beta 1 / 2$ kinase pathway metabolites (5). Additionally, when the levels of free radicals are elevated, amount of body anti-oxidant defense system markers such as TAC and SOD reduces (6). Moreover, prolonged oxidative stress induces the secretion of inflammatory factors such as C-reactive protein (CRP) and IL-18 (7-9). Sustained insulin resistance also leads to disturbed lipoprotein lipase activity and subsequent accumulation of free fatty acids (FFAs) which could interfere with various lipoproteins metabolism and thereby might contribute to dyslipidemia $(10,11)$. High appetite is another common symptom in T2DM patients which is associated with impaired hormonal balance that is 
characterized by high blood ghrelin and low leptin levels (12). These adverse clinical manifestations are responsible for further progression of DM and its unhealthy conditions $(11,13)$. Recently, genome-wide investigations have revealed the biological roles of several SNPs in the pathogens of DM (14). Since ApoB participates in the cellular cholesterol uptake from cholesterol-rich lipoproteins (15), variants in ApoB gene could predict hypercholesterolemia and subsequent oxidative and inflammatory states (16). On the other hand, the incidence of T2DM risk factors seems to be the result of the interaction between ApoB Ins/Del and EcoR1 SNPs and nutritional components (17). Dietary patterns as a principal aspect of life-style play important roles in the prevention as well as development of T2DM (18). Dietary phytochemical index (DPI) has shown negative relationships with oxidative stress and inflammation. DPI ranks persons according to their intakes of phytochemical rich foods such as whole grains, nuts, fruits and vegetables (19). Dietary quality index-international (DQI-I) and healthy eating index (HEI-2015) are among popular dietary indicators developed to assess the quality of the overall diet in different populations. DQI-I focuses on diversity, adequacy, balance and moderation of diet (20) and HEI-2015 aims at adhering to 2015-2020 dietary guidelines for Americans (2015-2020 DGA) (21). A number of studies have also suggested that adherence to healthy diet presented by high scores of DQI-I and HEI2015 is negatively associated with hemoglobin A1C (HA1C), CVD, gestational diabetes mellitus (GDM) and all-cause mortality in different populations $(22,23)$. In this study we aimed to determine the interaction between ApoB Ins/Del and EcoR1 polymorphisms and dietary quality indices including DQI-I, HEI-2015 and DPI on risk factors of cardio-metabolic diseases including dyslipidemia, inflammatory status, oxidative stress, obesity and hormonal abnormalities in patients with T2DM.

\section{Methods}

\subsection{Study design and subjects}

We conducted an observational cross-sectional study on patients with T2DM. Sample size calculating was through following formula:

$N=\left(\left(\left[(Z 1-a+Z 1-\beta) \times \sqrt{ } 1-r^{2}\right] / r\right)^{2}+2\right)$, When $r=0.15 \beta=0.95$ and $a=0.05$, the $N$ was equal to 694 . Due to data availability, we recruited 700 participants. Subjects were recruited by multistage cluster random sampling method from individuals who visited diabetes referral centers in different regions of Tehran, Iran during June 2011 to October 2012. Subjects were eligible for participation if they were adult and had been previously diagnosed with T2DM based on the decision of the endocrinologist. We excluded pregnant and lactating women and patients who had a current or a previous 2 months history of malignancies, abnormalities in kidney, hepatic, thyroid and cardiovascular system, alcohol intake, dependency on cigarette or drug, consumption of anti-inflammatory medications or dietary supplements. Additionally, subjects with an ongoing insulin therapy were not allowed to enter this research. There was no source of bias in present research. All stages of this study had been previously approved by the Ethics Committee of Tehran University of Medical Sciences (Ethnic number: IR.TUMS.VCR.REC.1395.15060). In 
addition, all eligible individuals signed a declaration on consent to participate after they were informed about aims and protocol of the study.

\subsection{Assessment of sociodemographic, anthropometric and physical activity variables}

Eligible participants were answered the questionnaires regarding their sociodemographic information such as age, job, medical history, duration and family history of T2DM, smoking and alcohol usage and consumption of lipid lowering medications. Assessment of physical activity was done using a validated questionnaire that was based on metabolic equivalent to task (MET-h/day) of daily activities (24). Weight $(\mathrm{kg})$ and height $(\mathrm{m})$ were measured via Seca falcon scales (Seca, Germany) by standard protocols. The determination of the waist circumference (WC) $(\mathrm{cm})$ was at the thinnest area of the waist and via a nonstretchable standard tape. Body mass index (BMI) was calculated via the following formula: weight/height ${ }^{2}\left(\mathrm{~kg} / \mathrm{m}^{2}\right)$.

2.3. Assessment of healthy eating index (HEl-2015), dietary quality index international (DQI-I) and dietary phytochemical index (DPI)

A validated food frequency questionnaire (FFQ) was used for estimation of the frequency of the consumption of 147 food components on a daily, weekly, monthly or annual basis (25). The detailed structures of HEI-2015, DQI-I and DPI have been numerously described elsewhere $(20,21,26)$. Healthy eating index-2015 (HEI-2015) is a new tool for prediction of the adherence level to 2015-2020 DGA. In brief, for total HEI-2015 score calculation, FFQ items were classified in to 13 food groups including 1) Total Vegetables, 2) Dairy, 3) Whole Fruits, 4) Total Fruits, 5) Total Protein Foods, 6) Greens and Beans, 7) Seafood and Plant Proteins, 8) Whole Grains, 9) Refined Grains, 10) Added Sugars, 11) Saturated Fats, 12) Fatty Acids and 13) Sodium. In this classification, there were similar score ranges $(0-5)$ for all parameters except for number $1-6$ food groups which had a score range between $1-10$. Scores of 13 food parameters were added to obtain the final HEI-2015 score in which 0 and 100 were indicative of minimum and maximum adherence to 2015-2020 DGA respectively. In DQI-I score, variety component (total score: $0-20$ ) composed of overall diversity between food groups (scores: $0-15)$ and diversity within protein food sources (scores: $0-5$ ). In adequacy component (total score: $0-40$ ), there were scores ranging from $0-5$ for investigation of sufficient intakes of 8 parameters including fruits, grains, fiber, protein, iron, calcium and vitamin C. Moderation component (total score: $0-30$ ) investigated restraint intake of 5 parameters (each of them had scores ranging from $0-6$ ) including total fat, saturated fat, sodium, cholesterol and empty calorie food. Overall balance (total score: $0-10$ ) was also according to the proper ratio of macronutrient intake including fat, carbohydrate and protein (score 0-6) and fatty acids intake including saturated fatty acids (SFA), polyunsaturated fatty acids (PUFA) and monounsaturated fatty acids (MUFA) (score $0-4$ ). Scores of four aspects were summed up to obtain the total score of DQI-I, in which score 0 showed minimal diet quality, while score 100 was indicative of maximal diet quality. The DPI for each subject was calculated as: the total calorie of all phytochemical rich food components 
(including whole grains, fruits, vegetables, natural fruit and vegetable juices, soy products, tomato sauces, nuts, legumes, olive and olive oil) divided by total calorie intake.

\subsection{Assessment of genetic and biochemical parameters}

Blood sampling carried out for all subjects after overnight fasting (8-12 hours). Afterwards, blood samples were undergone either centrifugation or PCR analysis. The detailed description of PCR, TaqMan assay method for evaluation of ApoB Ins/Deland EcoR1 SNPs have been published in our previous study (27). The centrifugation was conducted for measurement of biochemical markers in the serum.

Enzymatic method by commercially existing kits was used for determination of serum TC and triglyceride (TG) levels (Pars Azmoon, Iran), serum leptin (Bioassay Technology Co, China) and ghrelin (Bioassay Technology Co, Mediagnost, Germany) levels and serum IL-18, 8-iso-PGF2a and pentraxin-3 (PTX-3) levels (Shanghai Crystal Day Biotech Co., Ltd). Turbidimetry by a Roche Hitachi analyzer (Roche, Germany) was used for serum high density lipoprotein (HDL) and low density lipoprotein (LDL) levels determinations. Spectrophotometry procedure for serum TAC levels measurement was applied. Colorimetric procedure was used (Cayman Chemical Company, USA) for serum SOD levels measurements.

\subsection{Statistical analysis}

The Kolmogorov-Smirnov (K-S) test was used to decide whether variables in population had a normal distribution. Transformations to square or logarithmic were used for abnormal data. There were three genotypes of ApoB Ins/Del SNP including Ins/Ins, Ins/Del and Del/Del. The genotypes of Apo B EcoR1 SNP included $E+E+, E+E$-and $E$-E-. Independent t-test was applied for comparison of study quantitative outcomes between Del allele carriers and subjects with Ins/Ins homozygous and between $E$-allele carriers and subjects with $E+E+$ homozygous. HEI-2015, DQI-I and DPI scores were divided in to 3 equal intervals (tertile). ANOVA analysis test was carried out in order to compare study outcomes among groups of dietary indices. Finally, for the investigation of any interaction between dietary indices and mentioned polymorphisms on study outcomes, multivariate generalized linear model (GLM) test was applied. In all stages of our research's analysis, IBM SPSS (SPSS Inc., Chicago, IL, USA, version 21) was used, in which a p-value lower than 0.05 and 0.08 was assumed as significant and borderline significant, respectively.

\section{Results}

\subsection{Associations between DQI-I, HEI-2015 and DPI with cardio-metabolic parameters}

Table 1 provides the findings of ANOVA test for comparing cardio metabolic markers within the tertile of dietary indices in recruited subjects. We observed significant differences in HDL $(P=0.01)$ and LDL/HDL $(P=0.04)$ levels between HEl-2015 tertiles. When we compared the means of variables in paired groups, there were meaningful higher levels of HDL and LDL/HDL in tertile 1 and 3 than tertile 2 of HEI-2015 (For both variables: $P=0.02$ for tertile 1 and $P<0.01$ for tertile 3 ). Our analysis did not display anymore 
significant or borderline significant difference in study's variables within tertiles of DQI-I, HEI-2015 and DPI $(P>0.05)$. 
Table 1

Assessment of the associations of DQI-I, HEI-2015 and DPI with cardio metabolic parameters.

\begin{tabular}{|c|c|c|c|c|}
\hline Variable & Tertile 1 & Tertile 2 & Tertile 3 & P-value* \\
\hline \multicolumn{5}{|l|}{ DQI-I } \\
\hline BMI $\left(\mathrm{kg} / \mathrm{m}^{2}\right)$ & $29.70 \pm 4.97$ & $29.32 \pm 4.60$ & $28.92 \pm 4.39$ & 0.21 \\
\hline WC (cm) & $92.42 \pm 11.35$ & $92.46 \pm 9.92$ & $91.39 \pm 10.71$ & 0.51 \\
\hline LDL (mg/dl) & $108.27 \pm 32.52$ & $107.69 \pm 32.54$ & $110.59 \pm 38.58$ & 0.66 \\
\hline HDL (mg/dl) & $53.08 \pm 11.81$ & $52.05 \pm 11.09$ & $53.22 \pm 11.88$ & 0.53 \\
\hline LDL/HDL & $2.09 \pm 0.65$ & $3.00 \pm 12.25$ & $2.98 \pm 12.64$ & 0.53 \\
\hline $\mathrm{TC}(\mathrm{mg} / \mathrm{dl})$ & $195.18 \pm 59.49$ & $193.15 \pm 58.22$ & $191.84 \pm 74.36$ & 0.85 \\
\hline TG (mg/dl) & $175.40 \pm 95.57$ & $171.22 \pm 93.28$ & $177.33 \pm 107.00$ & 0.81 \\
\hline Leptin (ng/ml) & $25.75 \pm 11.87$ & $26.74 \pm 17.23$ & $24.44 \pm 14.78$ & 0.63 \\
\hline Ghrelin (ng/ml) & $2.28 \pm 1.27$ & $2.23 \pm 1.23$ & $2.63 \pm 1.53$ & 0.15 \\
\hline $\mathrm{CRP}(\mathrm{mg} / \mathrm{l})$ & $1.81 \pm 1.42$ & $2.26 \pm 1.52$ & $2.50 \pm 1.52$ & 0.08 \\
\hline IL-18 (pg/ml) & $255.16 \pm 28.85$ & $247.05 \pm 29.00$ & $246.09 \pm 28.00$ & 0.22 \\
\hline PTX-3 (ng/ml) & $2.61 \pm 0.39$ & $2.66 \pm 0.49$ & $2.64 \pm 0.51$ & 0.87 \\
\hline TAC (g/dl) & $2.53 \pm 0.61$ & $2.43 \pm 0.56$ & $2.43 \pm 0.49$ & 0.59 \\
\hline SOD (U/ml) & $0.148 \pm 0.04$ & $0.144 \pm 0.04$ & $0.140 \pm 0.04$ & 0.70 \\
\hline 8-iso-PGF2 a (pg/ml) & $72.66 \pm 5.46$ & $71.96 \pm 7.27$ & $73.10 \pm 5.97$ & 0.65 \\
\hline \multicolumn{5}{|l|}{ HEI-2015 } \\
\hline BMI $\left(\mathrm{kg} / \mathrm{m}^{2}\right)$ & $29.74 \pm 4.65$ & $29.36 \pm 4.91$ & $28.92 \pm 4.45$ & 0.18 \\
\hline WC (cm) & $92.85 \pm 10.93$ & $92.29 \pm 10.31$ & $91.21 \pm 10.86$ & 0.27 \\
\hline $\mathrm{LDL}(\mathrm{mg} / \mathrm{dl})$ & $107.49 \pm 33.95$ & $107.72 \pm 30.21$ & $111.87 \pm 39.62$ & 0.33 \\
\hline $\mathrm{HDL}(\mathrm{mg} / \mathrm{dl})$ & $53.50 \pm 11.57$ & $51.02 \pm 11.23$ & $54.12 \pm 12.04$ & 0.01 \\
\hline LDL/HDL & $2.05 \pm 0.63$ & $3.81 \pm 17.04$ & $2.11 \pm 0.70$ & 0.04 \\
\hline $\mathrm{TC}(\mathrm{mg} / \mathrm{dl})$ & $187.33 \pm 59.08$ & $197.67 \pm 58.36$ & $195.54 \pm 74.07$ & 0.20 \\
\hline
\end{tabular}

P-value*: ANOVA models. DQI-I: Dietary quality index-international, HEI-2015: Healthy eating index-U, DPI: Dietary phytochemical index, BMI: Body mass index, WC: waist circumference, LDL: Low density lipoprotein, HDL: High density lipoprotein, LDL/HDL: Low density lipoprotein / High density lipoprotein, TC: Total cholesterol, TG: Triglyceride, CRP: C-reactive protein, IL-18: Interleukin 18, PTX-3: Pentraxin-3, TAC: Total antioxidant capacity, SOD: super oxide dismutase and 8-iso-PGF2a: 8-isoProstaglandin F2a. 


\begin{tabular}{|c|c|c|c|c|}
\hline Variable & Tertile 1 & Tertile 2 & Tertile 3 & P-value* \\
\hline TG (mg/dl) & $173.97 \pm 107.74$ & $178.61 \pm 100.57$ & $172.00 \pm 86.69$ & 0.77 \\
\hline Leptin (ng/ml) & $24.42 \pm 13.56$ & $27.87 \pm 16.65$ & $24.27 \pm 13.67$ & 0.25 \\
\hline Ghrelin (ng/ml) & $2.33 \pm 1.33$ & $2.50 \pm 1.31$ & $2.37 \pm 1.46$ & 0.76 \\
\hline CRP (mg/l) & $1.93 \pm 1.50$ & $2.53 \pm 1.64$ & $2.10 \pm 1.34$ & 0.13 \\
\hline IL-18 (pg/ml) & $248.31 \pm 29.04$ & $250.67 \pm 26.69$ & $248.90 \pm 30.68$ & 0.91 \\
\hline PTX-3 (ng/ml) & $2.62 \pm 0.38$ & $2.62 \pm 0.47$ & $2.66 \pm 0.54$ & 0.87 \\
\hline TAC (g/dl) & $2.54 \pm 0.56$ & $2.50 \pm 0.58$ & $2.37 \pm 0.51$ & 0.27 \\
\hline SOD (U/ml) & $0.156 \pm 0.05$ & $0.138 \pm 0.04$ & $0.140 \pm 0.03$ & 0.12 \\
\hline 8-iso-PGF2 a (pg/ml) & $72.22 \pm 6.23$ & $72.47 \pm 6.36$ & $73.06 \pm 6.20$ & 0.78 \\
\hline \multicolumn{5}{|l|}{ DPI } \\
\hline BMI $\left(\mathrm{kg} / \mathrm{m}^{2}\right)$ & $29.59 \pm 4.96$ & $29.26 \pm 4.52$ & $29.17 \pm 4.56$ & 0.62 \\
\hline WC (cm) & $92.93 \pm 10.71$ & $91.76 \pm 10.24$ & $91.67 \pm 11.17$ & 0.40 \\
\hline LDL ( $\mathrm{mg} / \mathrm{dl})$ & $110.85 \pm 34.55$ & $105.47 \pm 33.64$ & $110.88 \pm 36.18$ & 0.17 \\
\hline $\mathrm{HDL}(\mathrm{mg} / \mathrm{dl})$ & $52.96 \pm 11.96$ & $53.58 \pm 12.37$ & $52.12 \pm 10.64$ & 0.42 \\
\hline LDL/HDL & $2.14 \pm 0.63$ & $2.79 \pm 11.51$ & $3.01 \pm 12.46$ & 0.63 \\
\hline $\mathrm{TC}(\mathrm{mg} / \mathrm{dl})$ & $200.09 \pm 80.18$ & $192.95 \pm 57.83$ & $187.64 \pm 51.64$ & 0.13 \\
\hline TG (mg/dl) & $189.41 \pm 117.74$ & $166.19 \pm 78.56$ & $169.38 \pm 95.01$ & 0.12 \\
\hline Leptin (ng/ml) & $23.92 \pm 13.42$ & $26.79 \pm 16.49$ & $25.34 \pm 13.73$ & 0.52 \\
\hline Ghrelin (ng/ml) & $2.39 \pm 1.60$ & $2.30 \pm 1.23$ & $2.49 \pm 1.31$ & 0.69 \\
\hline CRP (mg/l) & $2.16 \pm 1.42$ & $2.20 \pm 1.64$ & $2.23 \pm 1.47$ & 0.96 \\
\hline IL-18 (pg/ml) & $248.60 \pm 28.77$ & $249.67 \pm 30.91$ & $249.64 \pm 26.83$ & 0.97 \\
\hline PTX-3 (ng/ml) & $2.62 \pm 0.44$ & $2.72 \pm 0.48$ & $2.57 \pm 0.47$ & 0.23 \\
\hline TAC (g/dl) & $2.39 \pm 0.50$ & $2.46 \pm 0.55$ & $2.53 \pm 0.59$ & 0.45 \\
\hline SOD (U/ml) & $0.146 \pm 0.04$ & $0.146 \pm 0.05$ & $0.140 \pm 0.04$ & 0.76 \\
\hline
\end{tabular}

P-value*: ANOVA models. DQI-I: Dietary quality index-international, HEI-2015: Healthy eating index-U, DPI: Dietary phytochemical index, BMI: Body mass index, WC: waist circumference, LDL: Low density lipoprotein, HDL: High density lipoprotein, LDL/HDL: Low density lipoprotein / High density lipoprotein, TC: Total cholesterol, TG: Triglyceride, CRP: C-reactive protein, IL-18: Interleukin 18, PTX-3: Pentraxin-3, TAC: Total antioxidant capacity, SOD: super oxide dismutase and 8-iso-PGF2a: 8-isoProstaglandin F2a. 


\begin{tabular}{|c|c|c|c|c|}
\hline Variable & Tertile 1 & Tertile 2 & Tertile 3 & P-value* \\
\hline 8-iso-PGF2 a (pg/ml) & $72.37 \pm 5.85$ & $72.61 \pm 6.20$ & $72.79 \pm 6.68$ & 0.94 \\
\hline \multicolumn{5}{|c|}{$\begin{array}{l}\text { P-value*: ANOVA models. DQI--: Dietary quality index-international, HEI-2015: Healthy eating index-U, } \\
\text { DPI: Dietary phytochemical index, BMI: Body mass index, WC: waist circumference, LDL: Low density } \\
\text { lipoprotein, HDL: High density lipoprotein, LDL/HDL: Low density lipoprotein / High density } \\
\text { lipoprotein, TC: Total cholesterol, TG: Triglyceride, CRP: C-reactive protein, IL-18: Interleukin 18, PTX-3: } \\
\text { Pentraxin-3, TAC: Total antioxidant capacity, SOD: super oxide dismutase and 8-iso-PGF2a: 8-iso- } \\
\text { Prostaglandin F2a. }\end{array}$} \\
\hline
\end{tabular}

\subsection{Associations between ApoB Ins/Del and EcoR1 SNP and cardio-metabolic parameters}

The comparison of risk factors of cardio-metabolic variables within genotypes of ApoB Ins/Deland EcoR1 SNPs are presented in Table 2. A significant greater level of HDL was observed in $E$-allele carriers than subjects with $E+E+$ homozygous $(P=0.001)$. No significant association was discovered between other study outcomes and investigated polymorphisms $(P>0.05)$. 
Table 2

Assessment of the associations of ApoB Ins/Del and EcoR1 polymorphisms with cardio metabolic parameters.

\begin{tabular}{|c|c|c|c|c|c|c|}
\hline \multirow[b]{2}{*}{ Variable } & \multicolumn{3}{|c|}{ Ins/Del polymorphism } & \multicolumn{3}{|c|}{ EcoR1 polymorphism } \\
\hline & $\begin{array}{l}\text { Ins/Del and } \\
\text { Del/Del }\end{array}$ & Ins/Ins & $\begin{array}{l}\mathrm{P} \text { - } \\
\text { value }\end{array}$ & $\begin{array}{l}\mathrm{E}-/ \mathrm{E}+\text { and } \\
\mathrm{E}-/ \mathrm{E}-\end{array}$ & $\mathrm{E}+/ \mathrm{E}+$ & $\begin{array}{l}\text { P- } \\
\text { value }\end{array}$ \\
\hline BMI $\left(\mathrm{kg} / \mathrm{m}^{2}\right)$ & $29.18 \pm 4.31$ & $\begin{array}{l}29.42 \pm \\
4.86\end{array}$ & 0.40 & $30.28 \pm 4.99$ & $28.90 \pm 4.48$ & 0.16 \\
\hline WC (cm) & $92.70 \pm 10.90$ & $\begin{array}{l}91.46 \pm \\
10.01\end{array}$ & 0.21 & $\begin{array}{l}94.29 \pm \\
11.95\end{array}$ & $\begin{array}{l}91.33 \pm \\
10.23\end{array}$ & 0.20 \\
\hline LDL (mg/dl) & $113.01 \pm 36.35$ & $\begin{array}{l}107.02 \pm \\
33.91\end{array}$ & 0.71 & $\begin{array}{l}108.57 \pm \\
32.63\end{array}$ & $\begin{array}{l}108.01 \pm \\
36.28\end{array}$ & 0.20 \\
\hline HDL (mg/dl) & $53.40 \pm 11.78$ & $\begin{array}{l}52.63 \pm \\
11.63\end{array}$ & 0.83 & $\begin{array}{l}55.80 \pm \\
15.73\end{array}$ & $\begin{array}{l}52.72 \pm \\
11.61\end{array}$ & 0.001 \\
\hline LDL/HDL & $3.00 \pm 12.41$ & $2.47 \pm 8.23$ & 0.35 & $3.39 \pm 15.84$ & $2.46 \pm 7.93$ & 0.08 \\
\hline $\mathrm{TC}(\mathrm{mg} / \mathrm{dl})$ & $198.99 \pm 70.10$ & $\begin{array}{l}190.73 \pm \\
61.13\end{array}$ & 0.001 & $\begin{array}{l}202.77 \pm \\
82.58\end{array}$ & $\begin{array}{l}200.92 \pm \\
75.37\end{array}$ & 0.50 \\
\hline TG (mg/dl) & $175.82 \pm 96.16$ & $\begin{array}{l}174.30 \pm \\
99.82\end{array}$ & 0.41 & $\begin{array}{l}179.82 \pm \\
90.72\end{array}$ & $\begin{array}{l}193.28 \pm \\
114.77\end{array}$ & 0.18 \\
\hline Leptin (ng/ml) & $27.04 \pm 15.97$ & $\begin{array}{l}24.69 \pm \\
13.97\end{array}$ & 0.46 & $\begin{array}{l}26.12 \pm \\
15.16\end{array}$ & $\begin{array}{l}24.32 \pm \\
14.00\end{array}$ & 0.93 \\
\hline Ghrelin (ng/ml) & $2.27 \pm 1.25$ & $2.46 \pm 1.42$ & 0.17 & $2.39 \pm 1.16$ & $2.08 \pm 1.15$ & 0.94 \\
\hline CRP (mg/l) & $2.20 \pm 1.50$ & $2.20 \pm 1.52$ & 0.86 & $2.46 \pm 1.37$ & $2.29 \pm 1.53$ & 0.16 \\
\hline IL-18 (pg/ml) & $251.67 \pm 28.22$ & $\begin{array}{l}248.07 \pm \\
29.01\end{array}$ & 0.57 & $\begin{array}{l}251.48 \pm \\
25.20\end{array}$ & $\begin{array}{l}246.64 \pm \\
32.27\end{array}$ & 0.13 \\
\hline $\begin{array}{l}\text { Pentraxin } 3 \\
\text { (ng/ml) }\end{array}$ & $2.62 \pm 0.46$ & $2.64 \pm 0.47$ & 0.40 & $2.51 \pm 0.44$ & $2.66 \pm 0.48$ & 0.81 \\
\hline TAC (g/dl) & $2.35 \pm 0.52$ & $2.52 \pm 0.58$ & 0.77 & $2.53 \pm 0.60$ & $2.61 \pm 0.56$ & 0.91 \\
\hline SOD (U/ml) & $0.145 \pm 0.04$ & $\begin{array}{l}0.143 \pm \\
0.04\end{array}$ & 0.71 & $0.149 \pm 0.04$ & $0.142 \pm 0.04$ & 0.88 \\
\hline PGF2 a (pg/ml) & $72.02 \pm 5.95$ & $\begin{array}{l}72.92 \pm \\
6.39\end{array}$ & 0.41 & $72.90 \pm 6.17$ & $72.35 \pm 5.99$ & 0.68 \\
\hline $\begin{array}{l}\text { P-value*: Indepe } \\
\text { density lipoprote } \\
\text { lipoprotein, TC: T } \\
\text { Pentraxin-3, TAC } \\
\text { Prostaglandin F2 }\end{array}$ & $\begin{array}{l}\text { ent T-test model } \\
\text { HDL: High dens } \\
\text { al cholesterol, T } \\
\text { otal antioxidant }\end{array}$ & $\begin{array}{l}\text { MI: Body ma } \\
\text { lipoprotein, L } \\
\text { riglyceride, C } \\
\text { bacity, SOD: }\end{array}$ & er oxic & $\begin{array}{l}\text { ive protein, IL- } \\
\text { dismutase an }\end{array}$ & $\begin{array}{l}\text { erence, LDL: L } \\
\text { rotein / High d } \\
\text { : Interleukin } 18 \\
\text { 3-iso-PGF2a: } 8\end{array}$ & $\begin{array}{l}\text { Isity } \\
\text { PTX-3: } \\
\text { o- }\end{array}$ \\
\hline
\end{tabular}




\subsection{Interactions between DQI-I, HEI-2015 and DPI and Apo B Ins/Del and EcoR1 SNP on cardio-metabolic parameters}

The results of the interactions between dietary indices and Apo B SNPs (Ins/Del and EcoR1) on study outcomes are reported in Supplemental Figs. 1a-g. As shown in Supplemental Fig. 1.a and 1.b, the interactions between DQI-I and Ins/Del polymorphism on levels of serum leptin $(P=0.02$ and $P=0.01$ in crude and adjusted models, respectively) and 8-iso-PGF2a ( $P=0.01$ for both models) were statically significant. We showed that serum leptin in Del allele carriers was near to significantly lower in tertile 2 than tertile 3 and $1(P=0.05)$. However, in Ins/Ins homozygous there was not very significant difference in leptin level within DQI-I tertiles $(P=0.56$ (. Moreover, we found a borderline significant lower 8-iso-PGF2a concentration in tertile 2 compared to tertile 1 and 3 in Ins/Ins genotype $(P=0.05)$. However, there were increasing trends in 8-iso-PGF2a level from tertile 1 and 3 toward tertile 2 in Del allele carriers $(P=0.18)$. Findings of this investigation also indicated an interaction between HEI-2015 and Ins/Del SNP on IL-18 $(P=0.03$, Fig. 1.c) which even remained significant after controlling of confounding factors $(P=0.02)$. There was higher IL-18 in tertile 2 than tertiles 1 and 3 of HEl-2015 in Ins/Ins genotype $(P=0.17)$. While in Del allele carriers, subjects had higher IL-18 in tertiles 1 and 3 of HEl-2015 than tertile $2(P=0.15)$. According to our analysis, in crude but not in adjusted model there was close to significant interaction between ApoB EcoR1 SNP and DQI-I on TAC in study population ( $P=0.06$, Fig. 1.d). Our analysis showed that TAC level increased within HEl-2015 tertiles in E- allele carriers $(P=0.12)$. On the other hand, there was not any difference in TAC between tertile of HEl-2015 in subjects with $E+E+$ genotype $(P=0.48)$. In addition, in crude model, there were close to meaningful interactions between EcoR1 SNP and HEl-2015 on serum leptin $(P=0.05)$ and SOD $(P=0.05)$ levels in the participants which disappeared in adjusted models $(P>0.05)$ (Fig. 1.e and Fig. 1.f). It was observed that, in ApoB EcoR1 SNP, although there was a reducing trend in leptin level from tertile 1 toward tertile 3 of HEl-2015 in E- allele group ( $P=0.11$, Fig. 1.e), there was only a mild increasing trend in $E+E+$ homozygous $(P=0.62$, Fig. 1.e). Furthermore, while there was a significant decreasing trend in SOD within HEI-2015 tertiles in E- allele carriers $(P=0.04$, Fig. 1.f $)$, the trend of leptin from tertile 1 toward tertile 3 in participants with $E+E+$ homozygous were increasing and not meaningful $(P=0.53$, Fig. 1.f). Moreover, we found a statically significant interaction between EcoR1 SNP and DPI on serum TC level $(P=0.03$, Fig. 1.g) which remained meaningful after controlling of confounding variables $(P>0.03)$. In fact, $T C$ concentrations had decreasing and increasing trends during tertiles of DPI in subjects with $E+E+(P=0.08)$ genotype and in $E$ - allele carriers $(P=0.22)$, respectively (Fig. 1.g). The analysis of multivariate GLM in both crude and adjusted models, did not indicate other significant or close to significant interactions between Apo B Ins/Del SNP and dietary quality indices on lipid profile, anthropometric variables, CRP, ghrelin, PTX-3, TAC and SOD and between Apo B EcoR1 SNP and dietary quality indices on anthropometric variables, TG, LDL, HDL, LDL/HDL, IL-18, CRP, ghrelin and PTX-3 (P>0.05).

\section{Discussion}

The present study indicated a significant interaction between ApoB Ins/De/SNP and HEl-2015 on serum IL-18 level. In subjects with Ins/Ins genotype, there were higher IL-18 levels in tertile 2 than those in tertiles 1 and 3 of HEl-2015. While the trend of the IL-18 level seemed to be inversed in Del-allele carries (i.e. 
subjects had higher IL-18 in the tertiles 1 and 3 of HEl-2015 than tertile 2). The most important conclusion from this observation was that, Del allele carriers could respond to healthy diet with higher IL-18 only when HEl-2015 score is moderate. It has been reported that high blood IL-18 levels rise CVDs risk (28). HEI-2015 focuses on main elements of a healthy diet such as limited consumption of SFA and added sugar as well as high intake of fruits, vegetables, nuts and whole grains which have inflammation reducing properties $(29,30)$. Moreover, HEI-2015 score has been shown to have significant negative relationships with inflammation and risk of CVDs (31). There is currently evidence that Ins/De/SNP is related to deletion of 3 amino acids (Ala-Leu-Ala) from ApoB gene which alters the normal formation of recognition sit of ApoB for LDL recipient (32). Del allele in this SNP may lead to dyslipidemia especially hypercholesterolemia (33). Some evidence indicates that hypercholesterolemia people like Del-allele carriers are more responsive to beneficial dietary changes than subjects with normal blood cholesterol level (34). The present study also provided the evidence that Del allele carriers of ApoB Ins/DelSNP had significantly higher TC level than Ins/Ins genotype. On the other hand, the scoring systems in indices such as HEl-2015 and DQI-I does not consider over-consumption of energy and some food groups such as refined grains $(20,21)$ which might explain the higher IL-18 level in tertile 3 of HEI-2015 than tertile 2 in Del allele carriers. According to our best knowledge to date, there is no research that has investigated the interaction between ApoB SNPs and dietary factors on IL-18 levels and other inflammatory cytokines. There are some interaction studies between lipid, SFA and cholesterol intakes and ApoB Ins/Del polymorphism on lipid profile that reported insignificant results on TC $(35,36)$. However, there was not significant interaction between Ins/Del and dietary indices on TC in our study. This inconsistency might be due to differences in study population and the interaction of other food parameters in dietary indices in this study with ApoB Ins/Del SNP. In our study, we also represented a significant interaction between DQI-I and Ins/De/ variants on serum leptin concentration in both crude and adjusted models. It was observed that, serum leptin in individuals with Ins/Ins genotype was lower in tertile 3 and 1 of DQII than tertile 2. Although in those with Del allele, leptin level was not very different within DQI-I tertiles. A dietary pattern with high DQI-I and HEl-2015 scores is rich in MUFAs, PUFAs and fiber, poor in SFA and sugar and has a suitable proportion of total fat intake which can inhibit hypertriglyceridemia (37-39). Low blood TG level can protect against leptin resistance via change in receptor signaling or metabolism of leptin (40). Secondarily, we explored a borderline significant interaction between HEl-2015 and EcoR1 SNP on leptin level which disappeared after controlling of confounding factors. There was a reducing trend in leptin concentration from tertile 1 toward tertile 3 of HEI-2015 in E-allele carriers, while there was only a mild increasing trend in subjects with $E+E+$ homozygous. EcoR1 SNP is related to the substitution of lysine for glutamic acid that alters the formation and tendency of recognition site for LDL receptor that might lead to hypercholesterolemia (41). It appears that subjects with Del allele in Ins/Del SNP and $E$-allele in ECOR1 SNP are more beneficially responsive to healthier diet than those with Ins/Ins and $E+E+$ homozygous. Leptin is an adipokine which plays important roles in energy homeostasis and satiety. But hyperleptinemia and leptin resistance might be predictive factors of CVDs risk (42). To our knowledge, only a study by Rafiee et al assessed the interaction between dietary components and ApoB SNP on blood leptin levels in patients with T2DM. In their study, in Del allele carriers with T2DM, higher intake of MUFA, PUFA, SFA and protein and lower intake of carbohydrates were related to lower serum leptin

Page $12 / 20$ 
concentration. However there was not any significant difference in subjects with Ins/Ins genotype (43). Another finding of our study was the significant interaction between ApoB ECoR1 polymorphism and DPI on serum TC concentrations in both crude and adjusted models. Based on our analysis, during tertiles of DPI in $E+E+$ homozygous and $E$-allele carriers, TC levels had decreasing and increasing trends, respectively, indicating that phytochemical components may reduce cholesterol level in diabetic patients homozygous for $E+$ allele through their anti-oxidative and anti-inflammatory characteristics $(19,44)$. Currently, there is no experimental or human study on the interaction between genetic profile and DPI on cardio metabolic parameters. However, some researched have demonstrated the reducing effect of DPI on inflammation, obesity and pre-diabetes $(19,45)$. Furthermore, the interaction between dietary patterns or components and ApoB SNPs on oxidative stress and anti-oxidative markers has not been investigated, yet. Present study indicated significant interaction between ApoB Ins/De/polymorphism and DQI-I on 8iso-PGF2 $a$ in both crude and adjusted models. In fact, in Ins/Ins homozygous, moderate DQI-I score (tertile 2) was associated with benefits for oxidative stress shown by lower 8-iso-PGF2a levels compared to high (tertile 3) and low (tertile 1) DQI-I scores. On the other hand, there was increasing trend in 8-isoPGF2a level from tertile 1 and 3 toward tertile 2 in Del allele carriers. Puchau B revealed a possible protective role of high DQII scores against oxidative stress in healthy subjects (46). Therefore, in diabetic patients carrying Del allele, DQI-I appeared to have the strongest impact on reducing the serum 8-isoPGF2a level, when it has the highest score due to high intake of antioxidant nutrients such as vitamin $E$, $\mathrm{C}$, selenium, fiber $(47,48)$. While, it seems that only a moderate score of DQI-I is enough to reduce 8-isoPGF2a level in subjects with Ins/Ins homozygous. Additionally, in subjects with Ins/Ins homozygous, high intakes of energy, refined grains and fruits in tertile 3 of DQI-I may have contributed to higher 8-iso-PGF2a compared to tertile 2. The change of tendency of $A p o B$ to $L D L$ recipients might explain variability in plasma 8-iso-PGF2a associations with DQI-I values between genotypes of Ins/De/ polymorphism. In crude models, we also observed borderline significant interactions between ApoB EcoR1 SNP and DQI-I on TAC level and between ApoB ECOR1 SNP and HEI-2015 on SOD level. There was an elevating impact of high DQI-I scores in serum TAC in E-allele carriers. However, our results indicated that the response of SOD level as a main marker of antioxidant defense system to HEl-2015 was inversed and its concentrations decreased within HEI-2015 tertiles in E-allele carriers in crude model. But these borderline significant interactions on TAC and SOD disappeared after adjusting for confounding variables including BMI, age and smoking or alcohol uses. Findings of the relationship between HEl and oxidative stress are very limited. Tow investigation have demonstrated that the HEI-2015 had a positive association with TAC and $\mathrm{HEI}-2010$ had an insignificant association with $\operatorname{SOD}(49,50)$. We further indicated that greater adherence to the HEl-2015 had favorable effects on serum HDL level and LDL/HDL ratio, respectively, which can be due to high intake of cardio protective micro-nutrients irrespective of differences in genotypes of $A p o B$ SNPs (45). In addition, serum HDL concentration was significantly higher in E- allele carriers in ApoB EcoR1 SNP than $E+E+$ homozygous. It should be noted that, high $\mathrm{HDL}$ level in $E+E+$ homozygous in the current study can't be interpreted as a cardio protective factor because some studies have indicated that genetic mutations that increase blood HDL level do not necessarily protects against CVDs (51). In this study, lack of causal interpretation due to cross-sectional design and measuring of ApoB serum level due to limited budget must be taken into consideration as limitations. However, our research is unique, 
because for the first time, we assessed the interaction between DQI-I, DPI and HEI-2015 with ApoB Ins/Del and EcoR1 SNPs on cardio-metabolic risk factors in patients with T2DM.

\section{Conclusion}

We found that in T2DM patients carrying Del allele in ApoB Ins/De/ SNP, moderate DQI-I values might reduce 8-iso-PGF2 $a$ and leptin as well as moderate HEI-2015 scores may be accompanied with reduction of IL-18. Additionally, we suspect that higher DPI in $E+E+$ genotype of ApoB EcoR1 is probably associated with lower serum TC in patients with T2DM. Further investigations are required to identify the exact underlying mechanisms of the observed interaction in this study.

\section{List Of Abbreviations}

T2DM: Type 2 diabetes mellitus, SNP: Single nucleotide polymorphisms, PCR: Polymerase chain reaction, DQI-I: Dietary quality index-international, HEI-2015: Healthy eating index-2015, DPI: dietary phytochemical index, FFQ: food frequency questionnaire, 8-iso-PGF2a: 8-iso-prostaglandin F2 a, TC: total cholesterol, HDL: high density lipoprotein, LDL: Iow density lipoprotein, TG: triglyceride, IL-18: interleukin-18, TAC: total antioxidant capacity, SOD: superoxide dismutase, PTX-3: pentraxin-3, ApoB: apolipoprotein B, AGEs: advanced glycation end-products, CRP: C-reactive protein, FFAs: free fatty acids, 2015-2020 DGA: 20152020 dietary guidelines for Americans, GDM: gestational diabetes mellitus, WC: waist circumference, BMl: Body mass index, MUFA: monounsaturated fatty acids, SFA: saturated fatty acids, PUFA: polyunsaturated fatty acids, MET-h/day: metabolic equivalent to task, Multivariate GLM: multivariate generalized linear model, K-S test: Kolmogorov-Smirnov test, CVDs: Cardiovascular diseases.

\section{Declarations}

\section{Ethics approval and Consent to participate}

Present investigation was according to the ethical standards laid down in the 1964 Declaration of Helsinki and was approved by the Ethics Committee of Tehran University of Medical Sciences, Tehran, Iran (with ID number: IR.TUMS.VCR.REC.1395.15060). The Participants completed a written consent prior of including into the study.

\section{Consent for publication}

Written consent forms were received by participants for publication of this research.

\section{Availability of data and material}

The datasets analysed during the current study are available in the article text. The more detailed data of this study could be available by contact with: Email address: Elmira.karimii1994@gmail.com 
None

\section{Funding}

Tehran University of Medical Sciences provided fund for this investigation (Grant No: 20414).

\section{Authors' contributions}

EK: Conceptualization; Investigation; Writing- Original draft. GS: Supervision; Validation and Resources. MR: Formal analysis; Software, Supervision; Writing - review \& editing. FK: Conceptualization, Supervision; Funding acquisition and Project administration. All authors read and approved the final manuscript.

\section{Acknowledgment}

The authors would like to thank Tehran University of Medical Sciences for fund provision (Grant No: 20414) as well as the participants in this investigation.

\section{References}

1. Association AD. Diagnosis and classification of diabetes mellitus. Diabetes Care. 2010;33(Supplement 1):62-S9.

2. Cho N, Shaw J, Karuranga S, Huang Y, da Rocha Fernandes J, Ohlrogge A, et al. IDF Diabetes Atlas: Global estimates of diabetes prevalence for 2017 and projections for 2045. Diabetes Res Clin Pract. 2018;138:271-81.

3. Furukawa S, Fujita T, Shimabukuro M, Iwaki M, Yamada Y, Nakajima Y, et al. Increased oxidative stress in obesity and its impact on metabolic syndrome. J Clin Investig. 2017;114(12):1752-61.

4. Badawi A, Klip A, Haddad P, Cole DE, Bailo BG, El-Sohemy A, et al. Type 2 diabetes mellitus and inflammation: Prospects for biomarkers of risk and nutritional intervention. Diabetes metabolic syndrome obesity: targets therapy. 2010;3:173.

5. Brownlee M. The pathobiology of diabetic complications: a unifying mechanism. diabetes. 2005;54(6):1615-25.

6. Arab Sadeghabadi Z, Abbasalipourkabir R, Mohseni R, Ziamajidi N. Investigation of oxidative stress markers and antioxidant enzymes activity in newly diagnosed type 2 diabetes patients and healthy subjects, association with IL-6 level. Journal of diabetes metabolic disorders. 2019;18(2):437-43.

7. Monnier L, Mas E, Ginet C, Michel F, Villon L, Cristol J-P, et al. Activation of oxidative stress by acute glucose fluctuations compared with sustained chronic hyperglycemia in patients with type 2 diabetes. Jama. 2006;295(14):1681-7.

8. Esposito K, Nappo F, Marfella R, Giugliano G, Giugliano F, Ciotola M, et al. Inflammatory cytokine concentrations are acutely increased by hyperglycemia in humans: role of oxidative stress. Circulation. 2002;106(16):2067-72. 
9. Aboutaleb N, Zarrati M, Cheshmazar E, Shoormasti RS, Razmpoosh E, Nasirinezhad F. Association between the circulating leptin levels and the biomarkers of oxidative stress and inflammation among Iranian overweight and obese adults. Medical journal of the Islamic Republic of Iran. 2017;31:81.

10. Coppack S, Evans R, Fisher R, Frayn K, Gibbons G, Humphreys S, et al. Adipose tissue metabolism in obesity: lipase action in vivo before and after a mixed meal. Metabolism-Clinical Experimental. 1992;41(3):264-72.

11. Garg A, Grundy SM. Management of dyslipidemia in NIDDM. Diabetes Care. 1990;13(2):153-69.

12. Zhang S, Zhang Q, Zhang L, Li C, Jiang H. Expression of ghrelin and leptin during the development of type 2 diabetes mellitus in a rat model. Mol Med Rep. 2012;7(1):223-8.

13. Roebuck KA. Oxidant stress regulation of IL-8 and ICAM-1 gene expression: differential activation and binding of the transcription factors AP-1 and NF-kappaB. Int J Mol Med. 1999;4(3):223-53.

14. Ali O. Genetics of type 2 diabetes. World journal of diabetes. 2013;4(4):114.

15. Blackhart B, Ludwig E, Pierotti V, Caiati L, Onasch M, Wallis S, et al. Structure of the human apolipoprotein B gene. J Biol Chem. 1986;261(33):15364-7.

16. Catapano AL, Pirillo A, Norata GD. Vascular inflammation and low-density lipoproteins: is cholesterol the link? A lesson from the clinical trials. Br J Pharmacol. 2017;174(22):3973-85.

17. Hixson J, McMahan C, McGill H Jr, Strong J. Apo B insertion/deletion polymorphisms are associated with atherosclerosis in young black but not young white males. Pathobiological Determinants of Atherosclerosis in Youth (PDAY) Research Group. Arteriosclerosis and thrombosis: a journal of vascular biology. 1992;12(9):1023-9.

18. Maghsoudi Z, Azadbakht L. How dietary patterns could have a role in prevention, progression, or management of diabetes mellitus? Review on the current evidence. Journal of research in medical sciences: the official journal of Isfahan University of Medical Sciences. 2012;17(7):694.

19. Vincent HK, Bourguignon CM, Taylor AG. Relationship of the dietary phytochemical index to weight gain, oxidative stress and inflammation in overweight young adults. Journal of human nutrition dietetics. 2010;23(1):20-9.

20. Kim S, Haines PS, Siega-Riz AM, Popkin BM. The Diet Quality Index-International (DQI-I) provides an effective tool for cross-national comparison of diet quality as illustrated by China and the United States. J Nutr. 2003;133(11):3476-84.

21. Krebs-Smith SM, Pannucci TE, Subar AF, Kirkpatrick SI, Lerman JL, Tooze JA, et al. Update of the healthy eating index: HEl-2015. Journal of the Academy of Nutrition Dietetics. 2018;118(9):1591602.

22. Shin MK, Kim YS, Kim JH, Kim SH, Kim Y. Dietary Patterns and Their Associations with the Diet Quality Index-International (DQI-I) in Korean Women with Gestational Diabetes Mellitus. Clinical nutrition research. 2015;4(4):216-24.

23. Hu EA, Steffen LM, Coresh J, Appel LJ, Rebholz CM. Adherence to the Healthy Eating Index-2015 and Other Dietary Patterns May Reduce Risk of Cardiovascular Disease, Cardiovascular Mortality, and AllCause Mortality. J Nutr. 2020;150(2):312-21. 
24. Klishadi R, Khosravi A, Famouri F, Sadeghi M, Shirani S. Assessment of physical activity of adolescents in Isfahan. Journal of Shahrekord University of Medical Sciences. 2001;3(2).

25. Mirmiran P, Esfahani FH, Mehrabi Y, Hedayati M, Azizi F. Reliability and relative validity of an FFQ for nutrients in the Tehran lipid and glucose study. Public Health Nutr. 2010;13(5):654-62.

26. McCarty MF. Proposal for a dietary "phytochemical index". Medical hypotheses. 2004;63(5):813-7.

27. Rafiee M, Sotoudeh G, Djalali M, Alvandi E, Eshraghian M, Sojoudi F, et al. Dietary $\omega-3$ polyunsaturated fatty acid intake modulates impact of Insertion/Deletion polymorphism of ApoB gene on obesity risk in type 2 diabetic patients. Nutrition (Burbank, Los Angeles County, Calif). 2016;32(10):1110-5.

28. Everett BM, Bansal S, Rifai N, Buring JE, Ridker PM. Interleukin-18 and the risk of future cardiovascular disease among initially healthy women. Atherosclerosis. 2009;202(1):282-8.

29. Calder PC, Albers R, Antoine JM, Blum S, Bourdet-Sicard R, Ferns GA, et al. Inflammatory disease processes and interactions with nutrition. Br J Nutr. 2009;101(Suppl 1):1-45.

30. Giugliano D, Ceriello A, Esposito K. The effects of diet on inflammation: emphasis on the metabolic syndrome. J Am Coll Cardiol. 2006;48(4):677-85.

31. Li J, Demirel A, Azuero A, McLain A, Yarar-Fisher C. The Relationship Between Healthy Eating Index2015 and Cardiometabolic Risk Factors in People with Long-Standing Spinal Cord Injury. Current Developments in Nutrition. 2020;4(Supplement_2):540-.

32. Boerwinkle $E$, Chan L. A three codon insertion/deletion polymorphism in the signal peptide region of the human apolipoprotein $B(A P O B)$ gene directly typed by the polymerase chain reaction. Nucleic acids research. 1989;17(10):4003.

33. Machado MO, Hirata MH, Bertolami MC, Hirata RD. Apo B gene haplotype is associated with lipid profile of higher risk for coronary heart disease in Caucasian Brazilian men. J Clin Lab Anal. 2001;15(1):19-24.

34. Rantala M, Rantala TT, Savolainen MJ, Friedlander Y, Kesäniemi YA. Apolipoprotein B gene polymorphisms and serum lipids: meta-analysis of the role of genetic variation in responsiveness to diet. Am J Clin Nutr. 2000;71(3):713-24.

35. Pajukanta PE, Valsta LM, Aro A, Pietinen P, Heliö T, Tikkanen MJ. The effects of the apolipoprotein B signal peptide (ins/del) and Xbal polymorphisms on plasma lipid responses to dietary change. Atherosclerosis. 1996;122(1):1-10.

36. Jemaa R, Mebazaa A, Fumeron F. Apolipoprotein B signal peptide polymorphism and plasma LDLcholesterol response to low-calorie diet. Int J Obes. 2004;28(7):902-5.

37. Julibert A, Bibiloni MdM, Bouzas C, Martínez-González M, Salas-Salvadó J, Corella D, et al. Total and subtypes of dietary fat intake and its association with components of the metabolic syndrome in a Mediterranean population at high cardiovascular risk. Nutrients. 2019;11(7):1493.

38. Hannon BA, Thompson SV, Edwards CG, Skinner SK, Niemiro GM, Burd NA, et al. Dietary fiber is independently related to blood triglycerides among adults with overweight and obesity. Current developments in nutrition. 2019;3(2):nzy094.

Page $17 / 20$ 
39. Kell KP, Cardel MI, Bohan Brown MM, Fernández JR. Added sugars in the diet are positively associated with diastolic blood pressure and triglycerides in children. Am J Clin Nutr. 2014;100(1):46-52.

40. Vasselli JR, Scarpace PJ, Harris RB, Banks WA. Dietary components in the development of leptin resistance. Oxford University Press; 2013.

41. Sharma R, Mahajan M, Singh B, Singh G, Singh P. Role of the APOB gene polymorphism (c. $12669 \mathrm{G}>$ A, p. Gln4154Lys) in coronary artery disease in the Indian Punjabi population. Balkan Journal of Medical Genetics. 2011;14(2):35-40.

42. Patel SB, Reams GP, Spear RM, Freeman RH, Villarreal D. Leptin: linking obesity, the metabolic syndrome, and cardiovascular disease. Curr Hypertens Rep. 2008;10(2):131-7.

43. Rafiee M, Sotoudeh G, Djalali M, Alvandi E, Eshraghian M, Javadi F, et al. The interaction between apolipoprotein $\mathrm{b}$ insertion/deletion polymorphism and macronutrient intake on lipid profile and serum leptin and ghrelin levels in type 2 diabetes mellitus patients. Eur J Nutr. 2019;58(3):1055-65.

44. McCarty MF. Proposal for a dietary "phytochemical index". Medical hypotheses. 2004;63(5):813-7.

45. Davoudi-Kiakalayeh A, Mohammadi R, Pourfathollah AA, Siery Z, Davoudi-Kiakalayeh S. Alloimmunization in thalassemia patients: New insight for healthcare. International journal of preventive medicine. 2017;8.

46. Puchau B, Zulet M, Echávarri A, Hermsdorff HH, Alfredo M. Dietary Total Antioxidant Capacity: A Novel Indicator of Diet Quality in Healthy Young Adults. J Am Coll Nutr. 2009;28:648-56.

47. Lobo V, Patil A, Phatak A, Chandra N. Free radicals, antioxidants and functional foods: Impact on human health. Pharmacogn Rev. 2010;4(8):118.

48. Saura-Calixto F. Dietary fiber as a carrier of dietary antioxidants: an essential physiological function. J Agric Food Chem. 2011;59(1):43-9.

49. Zanella P, August P, Alves F, Matte C, Guerini de Souza C. Association of Healthy Eating Index and oxidative stress in adolescent volleyball athletes and non-athletes. Nutrition. 2019;60:230.

50. Heidari N, Jabbari M, Babashahi M, Nabie R, Jafarabadi MA, Arefhosseini SR. The association between healthy eating index and serum antioxidant indices in patients with different degrees of cataract compared to healthy subjects: a case-control study. Nutrition \& Food Science. 2020.

51. Voight BF, Peloso GM, Orho-Melander M, Frikke-Schmidt R, Barbalic M, Jensen MK, et al. Plasma HDL cholesterol and risk of myocardial infarction: a mendelian randomisation study. Lancet. 2012;380(9841):572-80.

\section{Figures}




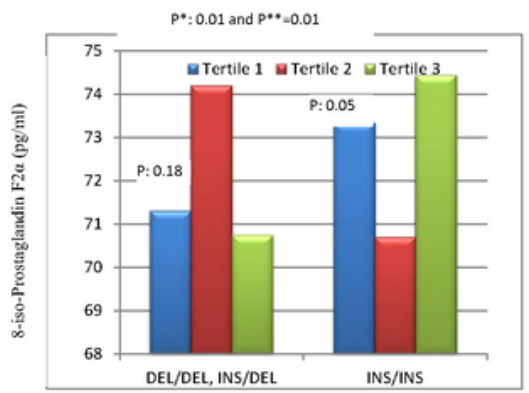

Fig-1.a

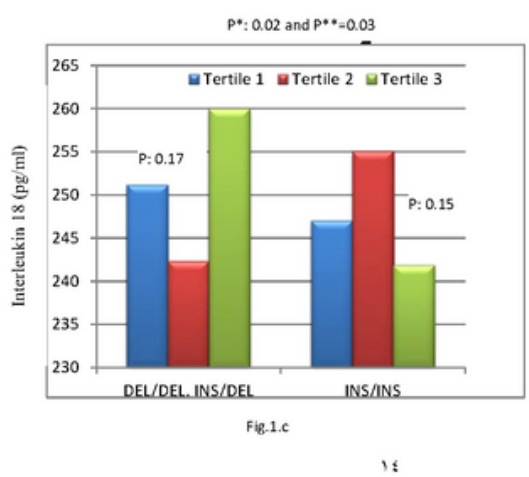

10

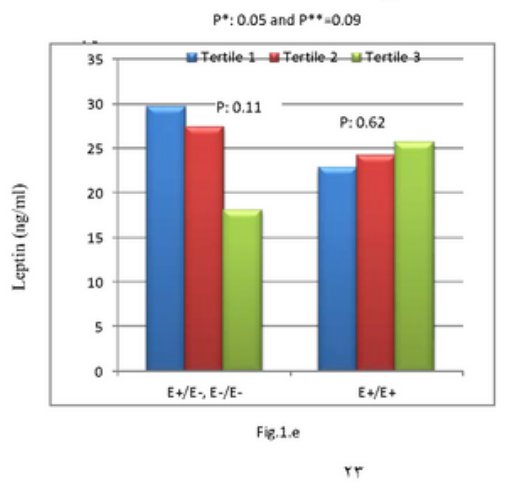

$p *: 0.03$ and $P *=0.03$

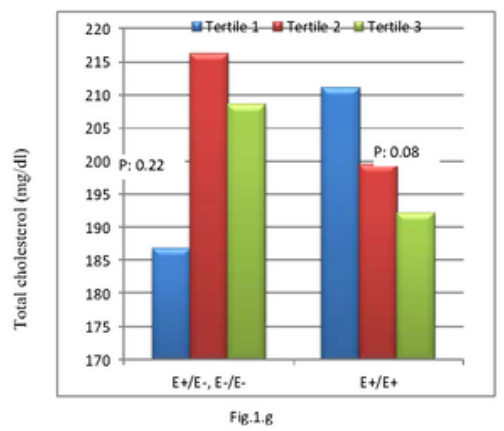

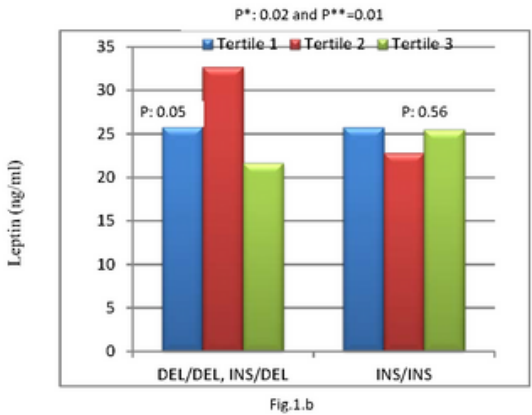
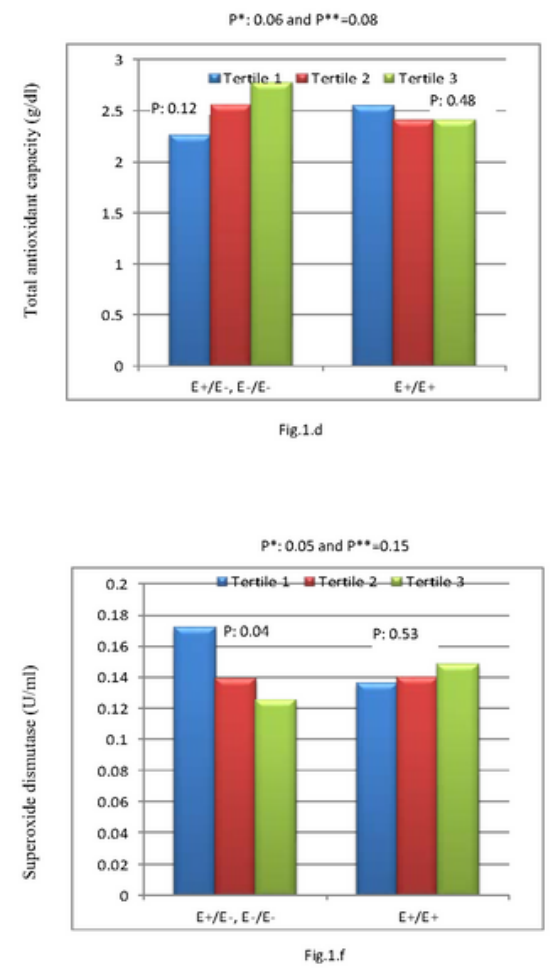

\section{Figure 1}

a: Interactions between Apo B INS/DEL and DQI-I on prostaglandin F2 a, Fig.1.b: Interactions between Apo B INS/DEL and DQI-I on leptin, Fig.1.c: Interactions between Apo B INS/DEL and HEI-2015 on IL-18, Fig.1.d: Interactions between Apo B EcoR1 and DQI-I on TAC, Fig.1.e: Interactions between Apo B EcoR1 and HEI-2015 on leptin, Fig.1.f: Interactions between Apo B EcoR1 and HEI-2015 on SOD, Fig.1.g: 
Interactions between Apo B EcoR1 and DPI on TC. ( $P^{*}=$ Crude mode, $\mathrm{P}^{\star *}$ : Adjusted model for age, gender, physical activity, and smoking and alcohol intake). Values are mean $\pm \mathrm{SE}$ 